\title{
Gehören wir dazu? Grenzziehungsprozesse und Positionierungen der zweiten Generation im Vergleich zwischen Luzern und Neuenburg
}

Kerstin Duemmler* und Janine Dahinden**

* Institut fédéral des hautes études en formation professionnelle (EHB IFFP IUFFP), CH-1000 Lausanne 16, kerstin.duemmler@iffp-suisse.ch.

** Laboratoire d'études transnationales, Université de Neuchâtel, CH-2000 Neuenburg, janine.dahinden@unine.ch.

\section{Abstracts \\ Gehören wir dazu? Grenzziehungsprozesse und Positionierungen der zweiten Generation im Vergleich zwischen Luzern und Neuenburg}

Statt wie üblich die Integrationsmodi der zweiten Generation zu untersuchen, nimmt der Artikel eine andere Perspektive ein und fragt, wie sich die zweite Generation gegenüber ethno-nationalen Grenzlinien positioniert und ob sie sich als zugehörig betrachtet. Anhand von Interviews mit 16 bis 19-Jährigen unterschiedlicher Herkunft aus den Kantonen Luzern und Neuenburg wird aufgedeckt, welche Positionierungen und Strategien sie angesichts von Grenzziehungen entwickeln. Der Vergleich macht insbesondere deutlich, wie die regionalen Kontexte ihre Positionierungen und Strategien kanalisieren. Unabhängig davon, ob sich die zweite Generation als zugehörig wahrnimmt, sind sie in Luzern durch Integrationsforderungen und in Neuenburg durch einen republikanischen Toleranzdiskurs geprägt.

Schlüsselwörter: Grenzen, Strategien, Zugehörigkeit, Integration, Ethnizität

Sentiments d'appartenances et positionnements vis-à-vis des frontières ethnonationales: une comparaison des jeunes de deuxième génération à Lucerne et à Neuchâtel

Cet article s'intéresse aux sentiments d'appartenance et aux positionnements des jeunes de deuxième génération vis-à-vis de certaines frontières symboliques ethno-nationales. Sur la base d'entretiens menés dans les cantons de Lucerne et Neuchâtel avec des jeunes de 16 à 19 ans d'origines différentes, nous mettons en lumière les stratégies et les discours qu'ils/elles développent par rapport à ces frontières. Nous montrons notamment comment le contexte institutionnel de chaque canton influence leurs discours et stratégies. Indépendamment de leur sentiment d'appartenance, les stratégies mises en œuvre par les jeunes sont marquées dans le canton de Lucerne par les exigences d'intégration et dans le canton de Neuchâtel par un discours républicain de tolérance.

Mots-clés: Frontières, stratégies, appartenance, intégration, ethnicité

\section{Do We Belong? Boundary Making and Positioning of the Second Generation: A Comparison between Lucerne and Neuchâtel}

Rather than to examine the modes of integration of the second generation as is usually done, the article chooses to ask how the second generation position themselves vis-à-vis certain ethno-national boundaries and whether they feel that they belong. Based on interviews with young people of different origins, aged from 16 to 19 years and residing in the cantons of Lucerne and Neuchâtel, we uncover their positionings and strategies when faced with boundaries. We notably show how the regional contexts influence their positions and strategies. Independently of their feelings of belonging, their strategies are strongly affected by the demands of integration in Lucerne and by a republican discourse of tolerance in Neuchâtel.

Keywords: Second generation, boundary strategies, belonging, integration, ethnicity 


\title{
Gehören wir dazu? Grenzziehungsprozesse und Positionierungen der zweiten Generation im Vergleich zwischen Luzern und Neuenburg
}

\author{
Kerstin Duemmler* und Janine Dahinden**
}

\section{$1 \quad$ Einleitung}

Die Migrationsforschung interessiert sich seit geraumer Zeit für die Integrationsprozesse der zweiten Generation ${ }^{1}$. Seit den 1990er Jahren sind zunächst in den USA revidierte Assimilationstheorien entstanden (Alba und Viktor 1997; Zhou 1997; Portes und Rumbaut 2005; Portes et al. 2009), wonach entgegen der ursprünglich linearen Assimilationsvorstellung mehrere Inkorporationswege für die zweite Generation existieren. Europäische Studien folgten diesem Trend und zeigten zudem, dass erhebliche Unterschiede innerhalb der gleichen Herkunftsgruppe existieren können und dass der nationale Kontext - Schulsysteme, öffentliche Diskurse, Arbeitsmarktstrukturen usw. - zentralen Einfluss auf die Integrationsprozesse hat (Bolzman et al. 2003; Simon 2003; Schneider und Crul 2010; Crul und Schneider 2013).

Dieser Artikel nimmt diese Thematik auf, schlägt aber eine andere Perspektive vor: Statt zu fragen, ob die zweite Generation sich strukturell und kulturell eingegliedert hat, interessiert uns, wie sie sich gegenüber Integrationsdiskursen positionieren und welche Strategien sie entwickeln. Unsere Fragestellung leitet sich aus drei Kritikpunkten ab, die gegenüber diesen (revidierten) Integrationstheorien formuliert wurden und die wir in unserer theoretischen Perspektive und Untersuchung aufnehmen.

Erstens wurde die nationalstaatlich und ethnisch zentrierte Epistemologie, die noch immer einen Grossteil der Integrationsforschung dominiert, von zahlreichen AutorInnen in Frage gestellt (Wimmer und Glick Schiller 2002; Dahinden 2008; Bommes und Thränhardt 2010; Amelina und Faist 2012; Dahinden 2012). Diese Epistemologie wird bspw. reproduziert, wenn Studien zur Integration der zwei-

\footnotetext{
* Institut fédéral des hautes études en formation professionnelle (EHB IFFP IUFFP), CH-1000 Lausanne 16, kerstin.duemmler@iffp-suisse.ch.

** Laboratoire d'études transnationales, Université de Neuchâtel, CH-2000 Neuenburg, janine. dahinden@unine.ch.

1 Der Begriff der zweiten Generation von MigrantInnen ist an und für sich problematisch, da eine essenzialisierte Sicht auf Migration mitschwingt. Es stellt sich die Frage, bei welcher Generation nicht mehr von MigrantInnen gesprochen und ab wann Kinder als zugehörig zu einem «Hier» betrachtet werden sollen. Als Alternative bietet sich an, von Einwandererkindern zu sprechen: Dieser Terminus stiftet allerdings Verwirrung, da wir in diesem Artikel empirisch mit Jugendlichen gearbeitet haben. Aus diesem Grunde verwenden wir den Begriff der zweiten Generation, mit allen Vorbehalten.
} 
ten Generation selbstredend verschiedene ethno-nationale Gruppen vergleichen. Zweitens kamen Integrationstheorien in die Kritik, politisch-normative Aspekte zu reproduzieren und dadurch nationalstaatliche Integrationsdiskurse zu unterstützen (Vasta 2007; Aumüller 2009), statt diese selbst zum Gegenstand der Forschung zu machen. Und drittens argumentieren wir, dass nicht nur nationale Kontexte unterschiedliche Inkorporationswege hervorbringen, sondern in der Schweiz kantonale Kontexte relevant sind.

Diese Kritikpunkte werden aufgenommen, indem die Positionierungen der zweiten Generation in diesem Artikel theoretisch unter einer Grenzziehungsperspektive (Lamont und Molnar 2002; Wimmer 2013) untersucht werden. Integration wird nicht als kulturelle oder strukturelle Anpassungsleistung verstanden. Gefragt wird vielmehr, wann sich welche ethno-nationalen Grenzlinien zwischen einem "Wir» und "den Anderen» etablieren oder auflösen und welche Positionen und Strategien die zweite Generation angesichts von Fremdzuschreibungen entwickelt. Der Artikel basiert auf einem empirischen Vergleich von Jugendlichen der zweiten Generation in den Kantonen Luzern und Neuenburg; Kontexte, die sich durch unterschiedliche Integrationsdiskurse und -politiken charakterisieren. Gezeigt wird, dass diese Kontexte nicht nur die symbolischen Grenzziehungen prägen, denen die zweite Generation gegenübersteht, sondern insbesondere auch die Strategien kanalisieren, wie mit Fremdzuschreibungen umgegangen wird.

In einem ersten Schritt legen wir die theoretischen Elemente der Grenzziehungsperspektive dar. Nach Präsentation der Methoden unserer empirischen Untersuchung gilt es die regionalen Spezifitäten der zwei Kantone im Hinblick auf die Diskurse über Integration aufzuzeigen. Schliesslich widmen wir uns den Positionierungen der zweiten Generation in Luzern und Neuenburg gegenüber den Integrationsdiskursen bzw. Grenzlinien. In der Diskussion halten wir fest, dass in den zwei Kontexten ähnliche Strategien auszumachen sind, wie mit Grenzziehungen umgegangen wird, allerdings sind diese Verhandlungen durch regionale kulturelle Repertoires und damit unterschiedliche Diskurse geprägt.

\section{Theoretischer Bezugsrahmen: die Grenzziehungsperspektive}

Wir schlagen vor, die Positionierungen der zweiten Generation gegenüber Integrationsdiskursen unter einer Grenzziehungsperspektive zu verstehen; d. h., wie sich essenzialistische ${ }^{2}$ Fremdzuschreibungen in die Selbstzuschreibungen der zweiten Generation einschreiben bzw. wie sie verhandelt werden können.

2 Unter Essenzialismus wird die Zuschreibung von Eigenschaften auf Personen verstanden, die unter eine spezifische Kategorie summiert werden, sodass die Personen als mit einer grundsätzlichen Essenz ausgestattet erscheinen (Phillips 2010) (z. B. alle Albaner hätten ungleiche Geschlechterbeziehungen). 
Ein relationales und prozessuales Verständnis von Ethnizität, wie es sich in der Tradition von Max Weber und Frederik Barth entwickelte, stellt eine nationalstaatlich und ethnisch zentrierte Epistemologie grundlegend in Frage. Während die Integrationsforschung häufig selbstverständlich von Grenzen zwischen ethnonationalen Kultursystemen ausgeht, untersuchen wir diese Grenzziehungsprozesse unmittelbar. Der Grenzziehungsansatz postuliert, dass nicht die kulturellen Unterschiede an sich, sondern der Bezug auf kulturelle Unterschiede zur Markierung und Rechtfertigung entscheidend ist, ob ethnische oder nationale Gruppen(grenzen) fortbestehen (Barth 1969; Brubaker et al. 2004; Wimmer 2008; Duemmler 2015). Kulturelle Eigenschaften, ob real oder erfunden, werden herangezogen um Zugehörigkeitsgefühle, Gruppenmitgliedschaft und Ausschluss anzuzeigen (Weber 1922 [1980]). Lamont und Molnar (2002, 168) definieren (symbolische) Grenzen als konzeptionelle Unterscheidungen, die soziale Akteure aufgreifen, um Unterschiede zwischen Individuen oder Gruppen hervorzubringen, aufrechtzuerhalten, aufzulösen, zu rechtfertigen oder anzufechten.

«Integration» wird unter dieser analytischen Sichtweise nicht als kulturelle und strukturelle Anpassungsleistung von Einwanderergruppen verstanden, erforscht wird stattdessen, wann und wie sich ethnische oder nationale Grenzziehungen zwischen einem «Wir» und den "Anderen» etablieren, überschritten werden oder auflösen, und welche Konsequenzen diese Grenzziehungsprozesse haben (Zolberg und Woon 1999; Alba 2005; Dahinden 2011).

Grenzziehungen variieren also je nach Kontext, da sie von den Verhandlungen und Kämpfen der sozialen Akteure um Kategorisierungen und Deutungen abhängen (Lamont und Molnar 2002; Neckel und Sutterlüty 2008). Für unsere Untersuchung heisst das auch, dass die Fremdzuschreibungen und Formen von Zugehörigkeit, die der zweiten Generation auferlegt werden, einen Einfluss auf ihre Selbstzuschreibungen und Positionierungen gegenüber den Grenzlinien haben (Jenkins 2008). Es handelt sich dabei um keinen neutralen Prozess, denn die involvierten Individuen und Gruppen erfahren durch die Klassifizierung in bestimmte Kategorien Wertschätzungen oder Missachtungen (Weber 1922 [1980]; Neckel und Sutterlüty 2008). Zudem sind Grenzziehungen in grössere Ungleichheits- und Machtverhältnisse eingebunden, denn welche Grenzlinien sich in einem Kontext durchsetzen, ist abhängig von den Ressourcen der Akteure, Zuschreibungen anderen aufzudrängen oder sich ihnen zu widersetzen (Elias und Scotson 1994 [1965]; Jenkins 2008).

Auch die kulturellen Repertoires (Swidler 1986), die zur Rechtfertigung von Grenzen eingesetzt werden, variieren (Lamont und Mizrachi 2012, 366 f.). Sie gewähren bzw. verwehren der zweiten Generation bestimmte Formen der Zugehörigkeit und beeinflussen dadurch ihre Positionierungen gegenüber Grenzziehungen. So stellt sich die Frage, wie sich die Kantone Luzern und Neuenburg bei der Institutionalisierung von Grenzlinien und kulturellen Repertoires zu ihrer Rechtfertigung unterscheiden. 
Der Grenzziehungsansatz liefert auch Strategien, wie sich Individuen gegenüber Zuschreibungen und Missachtungen positionieren und sie u. U. verhandeln können. Durch Grenzüberschreitungen (boundary crossing) versuchen Individuen die eigene Position im Hinblick auf die Grenzlinie und Hierarchie (z. B. Schweizer/Ausländer basierend auf wahrgenommenen Integrationsdefiziten) zu verbessern. Sie identifizieren sich mit der sozial wertgeschätzten Kategorie und versuchen sich anzupassen (Wimmer 2008, 1039f.). Durch Grenzverwischung (boundary blurring) wird die Grenzlinie durch die Betonung anderer Kriterien, bspw. universelle Zugehörigkeiten (wie Menschenrechte), für irrelevant erklärt. Was vorher als «anders» angesehen wurde, wird nun als «vertraut» erklärt, und gründet häufig auf einer formellen Anerkennung multipler Zugehörigkeiten (z. B. Zweisprachigkeit) (Zolberg und Woon 1999, 8 f.). Aufwertungsstrategien zielen darauf ab, die Hierarchie zwischen Kategorien umzudeuten und die eigene stigmatisierte Gruppe (auch auf Kosten anderer) aufzuwerten (Wimmer 2008, 1037f.). Zuletzt zielen Grenzverschiebungen (boundary shifting) auf eine Öffnung der Grenze und Erweiterung der Wir-Gruppe um die vormals ausgeschlossene Ihr-Gruppe ab (Zolberg und Woon 1999).

\section{Methoden}

Der Artikel stützt sich auf qualitative Daten, die im Rahmen eines grösseren Forschungsprojektes erhoben wurden. Die Studie nahm bewusst keine ethno-nationale Gruppe zum Ausgangspunkt, sondern interessierte sich für alltägliche Austauschbeziehungen zwischen Schweizer Jugendlichen und jungen Erwachsenen der zweiten Generation unterschiedlicher Herkunft im Alter von 16 bis 19 Jahren. Eine dreimonatige ethnografische Feldforschung ${ }^{3}$ fand in jeweils vier Luzerner und vier Neuenburger Schulen (je 3 Berufsschulen und 1 Gymnasium) statt. Wir stützen uns für diesen Artikel auf 24 Leitfadeninterviews mit Jugendlichen der zweiten Generation $^{4}$, wobei der Leitfaden flexibel eingesetzt wurde, um den Relevanzstrukturen der Jugendlichen gerecht zu werden (Charmaz 2001). Konkret gefragt wurde, welche Bedeutung nationale Herkunft und Religion im Alltagsleben hat (z. B. mit der Familie, beim Eingehen von Beziehungen) und wie Mitmenschen auf ihre Herkunft und Religion reagieren. Auf Integration oder andere Herkunftsgruppen wurde nur eingegangen, wenn dies von den Jugendlichen selbst angesprochen wurde.

Auf ethnografische Feldbeobachtungen kann hier nicht eingegangen werden, um dem regionalen Vergleich der zweiten Generation genug Raum zu geben. Duemmler (2015) zeigt allerdings anhand von Unterrichtsbeobachtungen, wie Integrationsvorstellungen in der Schule an die jungen Erwachsenen vermittelt und wie Grenzlinien im Schulalltag reproduziert bzw. infrage gestellt wurden.

$4 \quad$ Das heisst, ihre beiden Eltern sind in die Schweiz eingewandert, sie selbst sind nicht zwingend auch eingewandert, haben aber den grössten Teil ihrer Schulzeit in der Schweiz verbracht. 
Die Daten wurden mit Hilfe eines Codings analysiert (Glaser und Strauss 1967; Charmaz 2001), d. h., wir kodierten zunächst offen und induktiv, um nah am Interviewmaterial zu sein. Konkret interessierten wir uns dafür, welche Grenzlinien und ethnischen Fremdzuschreibungen die zweite Generation wahrnimmt und welche Selbstzuschreibungen in ihren Positionierungen gegenüber den Grenzlinien virulent werden. Anschliessend wurden ausgewählte Codes (z. B. Migranten sollen sich integrieren, Kultur als Bereicherung, Diskriminierungserfahrung) fokussierter kodiert, interpretiert und Zusammenhänge herausgearbeitet. Die Interviews wurden schliesslich anhand der Codes gruppiert, d.h., nach Gemeinsamkeiten und Unterschieden in den Wahrnehmungen, Handlungsmustern und Strategien der zweiten Generation gesucht und eine Ordnung (getrennt nach Kanton) geschaffen. Die Typisierung erfolgte idealtypisch im Sinne Webers; es wurde ein Konstrukt auf Basis mehrerer Fälle geschaffen (Kelle und Kluge 2010).

Die Herkunftsnationalitäten der zweiten Generation sind in Luzern und Neuenburg verschieden, da in den zwei Kantonen unterschiedliche Einwanderergruppen zu finden sind. Allerdings ist dies für unsere Untersuchung von untergeordneter Bedeutung, da wir uns weniger für spezifische ethno-nationale Gruppen interessieren, sondern allgemein für die Grenzziehungen und Positionierungen, unter Einwirkung der regionalen Kontexte. In Luzern haben sechs der 14 Jugendlichen Vorfahren aus dem Kosovo, die Eltern der übrigen 8 stammen aus Tschechien, Kroatien (bei zwei Jugendlichen), Montenegro, Ungarn, Serbien, Afghanistan und Portugal. Zehn Jugendliche besitzen die Schweizer Staatsbürgerschaft und vier haben eine Niederlassungsbewilligung. In Neuenburg haben drei der zehn Jugendlichen Vorfahren aus Italien, zwei aus Bosnien, zwei aus Portugal, die anderen Eltern stammen aus Somalia, Tunesien (und Frankreich) und Ile Maurice (und Italien). Sieben Jugendliche sind eingebürgert bzw. im Einbürgerungsverfahren, zwei haben jeweils eine Jahres- resp. Niederlassungsbewilligung.

\section{$4 \quad$ Integrationsdiskurse in den Kantonen Luzern und Neuenburg}

In Debatten über Assimilation und Integration wird definiert, welche Einwanderer zur nationalen Gemeinschaft dazugehören und mit welchen Argumenten diese Grenzlinie verteidigt wird. Solche Debatten haben den Stellenwert von «kulturellen Repertoires» (Swidler 1986), auf die sich Akteure in ihren Handlungen (oft unbewusst) beziehen können. In diesem Artikel erschliessen wir diese «kulturellen Repertoires» durch eine Analyse der Integrationsdokumente und -gesetze in den zwei Kantonen. Aus diesen Dokumenten und Gesetzen lassen sich stellvertretend «öffentliche Diskurse» herauskristallisieren, die das Ergebnis von Interessenskämpfen und Repräsentationen verschiedener Akteure sind. Wir gehen also davon aus, dass die Gesetze und Leitbilder öffentliche Anliegen und Repräsentationen widerspie- 
geln. Die Jugendlichen kennen diese Dokumente zwar nicht im Einzelnen, aber sie verwenden, wie später gezeigt wird, die spezifischen «kulturellen Repertoires» als Deutungsschemen in ihren Positionierungen gegenüber Grenzlinien. Andere Institutionen, die solche «kulturellen Repertoires» mitproduzieren, sind die Schulen (für dieses Forschungsprojet siehe Duemmler 2015 oder Schiffauer et al. 2002) oder bspw. auch die Medien.

Es werden deshalb an dieser Stelle die wichtigsten Elemente der Integrationsdiskurse und -politik für beide Kantone umrissen. Im Kanton Luzern gibt es zwar kein Integrationsgesetz, denn ein Gesetz zur Förderung des gesellschaftlichen Zusammenhaltes wurde 2010 in einer Volksabstimmung abgelehnt. Dennoch erliess der Regierungsrat 2001 ein Integrationsleitbild, das von Einwanderern eine integrative Eigenleistung fordert, ohne dass sie jedoch ihre Herkunftskultur vollkommen aufgeben müssten. Dazu heisst es:

Sie [Ausländer] erlangen grössere Anerkennung und auch mehr Chancengleichheit, wenn sie sich so gut wie möglich in die Kultur unseres Landes integrieren, ohne dass sie deswegen ihre Herkunft und die mitgebrachten kulturellen Werte und damit ihre Identität verleugnen müssen. (Regierungsrat 2001, 16)

Das Leitbild erwartet eine partielle kulturelle Anpassung seitens der Einwanderer und gründet dabei auf einem essenzialistischen Ethnizitätsverständnis, denn Kultur, Herkunft und Identität (mit Blick auf die Schweiz und das Herkunftsland) werden selbstverständlich gleichgesetzt. Kulturelle (und religiöse) Differenzen werden dennoch als Bereicherung angesehen (Regierungsrat 2001, 17) und ihnen sollte mit Respekt und Toleranz begegnet werden, wenn diese im Rahmen der Rechtsordnung gepflegt würden (Regierungsrat 2001, 19). Dieser Vorstoss hin zu einer Grenzöffnung zwischen Schweizern und Ausländern hat allerdings ein klares Limit. Das Leitbild spricht sich explizit gegen eine multikulturalistische Gesellschaft aus, d. h. ein gleichberechtigtes und friedliches Zusammenleben aller Kulturen (Regierungsrat 2001, 19) und warnt vor der «Bildung von Ausländerquartieren» und der «Aufsplitterung der Gesellschaft in (...) ethnische Gruppen mit nationalistischem Kulturbewusstsein» (Regierungsrat 2001, 20). Die im Kanton Luzern eher verhalten gebliebene Grenzöffnung gegenüber Ausländern kommt auch darin zum Ausdruck, dass das Leitbild dazu aufruft, AusländerInnen, «die aus anderen Kulturkreisen, zum Teil auch aus einem Klima der Gewalt kommen», besonders mit den «Regeln des Zusammenlebens» (Regierungsrat 2001, 28) vertraut zu machen.

Festgehalten werden kann, dass ein assimilatorisches Integrationsverständnis, auch wenn kulturelle Differenzen zum Teil als Bereicherung angesehen werden, im Kanton Luzern dominiert. So erstaunt auch nicht, dass der Kanton (im Gegensatz zu Neuenburg) mit nichteuropäischen Einwanderern Integrationsvereinbarungen 
und -empfehlungen abschliesst (Gesundheits- und Sozialdepartement 2013, 10), obwohl der Bund diesen Einsatz im Integrationsgesetz nicht vorschreibt.

Das Leitbild spricht sich darüber hinaus für den Respekt der Ausländer als Menschen sowie für die Chancengleichheit im Bildungs- und Erwerbssystem aus und wendet sich gegen Diskriminierung und Rassismus (Regierungsrat 2001, 18-19). Diese Grenzverwischung scheint allerdings bisher nur auf dem Papier zu existieren. Ein Blick in das Integrationsprogramm 2014-2017 zeigt, dass, wenngleich der Kanton bei der Integrationsförderung aktiv ist (z. B. Information über Rechte und Pflichten), es kaum Massnahmen im Bereich des Schutzes vor Diskriminierung gibt (Gesundheits- und Sozialdepartement 2013, 6).

Die Integrationsdiskurse und -politik in Neuenburg schreiben sich in ein anderes Register ein und verdeutlichen prägnant die interne Heterogenität der Schweiz. In Anlehnung an Brubaker (1992) findet sich in diesem französischsprachigen Kanton ein republikanisches Staatsbürgerschaftsmodell, welches grundsätzliche Differenzen zum ethnischen Modell aufweist, das im deutschsprachigen Kanton Luzern verankert ist.

Die Charte de la citoyenneté (Service de la cohésion multiculturelle 2009, 1) wird jedem/r neuen ZuzüglerIn im Kanton zugestellt und hält diese Prinzipien fest:

Le canton de Neuchâtel est un Etat qui garantit à ces habitants des libertés et droits fondamentaux (Etat libéral), un Etat dans lequel le peuple participe à la formation de la volonté et à l'exercice du pouvoir (Etat démocratique), un Etat qui accorde à ces concitoyens une certaine protection sociale (Etat social) (...) les ressortissants d'autres pays qui séjournent en Suisse sont soumis au même ordre juridique que les citoyens suisses. Ils n'ont cependant aucune obligation juridique, lorsqu'ils proviennent d'autres cultures, d'adapter leur façon de vivre à celle des Suisses. (...) Ainsi, si les peuples se caractérisent naturellement par leur diversité, c'est la tolérance, l'ouverture d'esprit, le respect et l'appréciation de la richesse et de la diversité des cultures qui peuvent assurer l'équilibre au sein des collectivités mixtes.

Aus dieser Charte de la citoyenneté lassen sich zwei Grunddiskurse herauslesen. Erstens eine republikanische Haltung gegenüber Staatsbürgerschaft. Diese wird dadurch bestätigt, dass Neuenburg AusländerInnen die Möglichkeit gibt, auf kantonaler Ebene zu wählen und auf Gemeindeebene gewählt zu werden, falls sie eine Niederlassungsbewilligung besitzen und seit mindestens 5 Jahren im Kanton (resp. $1 \mathrm{Jahr}$ in der Gemeinde) wohnen. In einer Volksabstimmung 2011 wurde dieses Recht für AusländerInnen in Luzern abgelehnt. Zweitens wird kulturelle Diversität zwar essenzialisiert, aber durchweg (im Gegensatz zu Luzern) positiv aufgeladen. Toleranz gegenüber kulturell anderen wird eingefordert und kulturelle Diversität wird als Bereicherung dargestellt. Dieser republikanische Toleranzdiskurs gegenüber kultureller Vielfalt verbindet Offenheit gegenüber «anderen Kulturen» mit der uni- 
versalistischen Idee, dass alle Menschen die gleichen Rechte und Pflichten haben (etwa politische Partizipation) und für alle die Menschenwürde gilt, unabhängig von ihrer Nationalität.

Das revidierte Integrationsgesetz aus dem Jahre $2013^{5}$ schreibt diese Ideen fort und legt im ersten Artikel seine Funktion dar:

Elle encourage (...) la pleine participation des personnes issues de la migration à la société, de façon plus générale, tend à promouvoir l'égalité des droits et devoirs ainsi que la non-discrimination pour tout un chacun dans les limites de la constitution et de la loi. (Loi sur l'intégration et la cohésion multiculturelle, art. 1)

Die republikanische Geisteshaltung wird hier fortgeschrieben, als dass ein grosses Gewicht auf Rechtsgleichheit, Nicht-Diskriminierung wie auch Partizipation gelegt wird. So erstaunt es nicht, sind im Integrationsprogramm des Kantons Neuenburg (Service de la cohésion multiculturelle 2013) im Gegensatz zu Luzern konkrete Massnahmen gegen Diskriminierung verankert.

Zusammenfassend kann festgehalten werden, dass sich in diesen zwei Kantonen widerspiegelt, was Menet und Wichmann $(2012,19)$ folgendermassen beschreiben: «Deutschschweizer Kantone [kombinieren] ein assimilatorisches Integrationsverständnis und die Förderung der Chancengleichheit. (...) [Französischsprachige] Kantone [setzen] auf eine Kombination der politischen Teilhabe mit der Förderung der Chancengleichheit». Luzern verfolgt die Integration der Einwanderer über einen essenzialistischen Kultur- und Ethnizitätsdiskurs. Neuenburg zeichnet sich hingegen nicht nur durch mehr Toleranz gegenüber kultureller Differenz (wenn auch essenzialisierter) aus, sondern gesteht in seinem Staatsbürgerschaftskonzept allen BürgerInnen auch gleiche Rechte und Pflichten zu. Das bedeutet, dass die Grenzlinie zwischen SchweizerInnen und AusländerInnen in Luzern über positiv, aber auch negativ aufgeladene kulturelle Differenzen etabliert wird. In Neuenburg ist diese Grenzlinie auch vorhanden, aber weniger ausschliessend, da die republikanische Staatsbürgeridee (Rechtsgleichheit, Partizipation, Nicht-Diskriminierung usw.) sie verschwimmen lässt. In Luzern werden solche Themen diskutiert, doch kaum umgesetzt, sodass die Grenzlinie klarer aufscheint.

Wie wir im nächsten Kapitel zeigen, sind diese regional spezifischen Ideen und «kulturellen Repertoires» die Skripte, entlang derer die Positionierung der Jugendlichen erfolgt. 


\section{Luzern: Integrationsforderungen in den Positionierungen der zweiten Generation}

Das «Leitmotiv», das die Interviews in Luzern mit der zweiten Generation durchzog - und zwar ohne, dass wir diese Aspekte eingebracht hätten -, war die Idee der Integrationsforderungen: Die Jugendlichen nahmen allesamt spontan auf dieses spezifische «kulturelle Repertoire» Bezug. Allerdings positionierten sich die Jugendlichen unterschiedlich, je nachdem wie sie die Grenzlinie zwischen Schweizern/ Ausländern und Stigmatisierungen deuteten. Die vier idealtypischen Positionierungen lassen sich mit den vier Strategien (Grenzüberschreitung, -verwischung, -öffnung durch Grenzziehung und Aufwertung) in Verbindung bringen ${ }^{6}$ und zeigen, dass die Grenzlinie vor allem seitens der Jugendlichen mit Vorfahren aus dem Kosovo (sie verwischen die Grenze nicht) als klar wahrgenommen wird. Dies überrascht kaum, werden sie doch in der Deutschschweiz am häufigsten mit Diskriminierungen konfrontiert (Fibbi et al. 2006).

\subsection{Individuelle Grenzüberschreitung (boundary crossing) und Integrationsforderung}

Kristin versteht sich ausschliesslich als Schweizerin und stellt sich völlig in Einklang mit assimilatorischen Integrationsforderungen; sie ist die einzige Vertreterin dieser Strategie der «individuellen Grenzüberschreitung». Sie berichtet, dass sie als Kind durch MitschülerInnen aufgrund ihrer kosovarischen Herkunft stigmatisiert und ausgegrenzt wurde. Dennoch ist sie überzeugt, dass Ausländer «einen schlechten Ruf» und Ausschluss zu verantworten hätten, weil sie oft nicht bereit seien, sich anzupassen. Im Interview äusserte sie sich über das Verhältnis zwischen Ausländern und Schweizern:

[I]ch habe auch das Gefühl, dass die Ausländer selber schuld sind, dass sie so einen schlechten Ruf haben. Da sie immer Schlägereien machen. (...) Aber wenn man älter ist, so mit 18, da kannst du dich ja auch anpassen. (...) Ich meine, ich habe jetzt Kollegen, ich habe mich anpassen können. So in der zweiten Klasse und als Kleine, das ist schon schlimm, wenn du als Aussenseiter bezeichnet wirst, nur weil du eine andere Nationalität hast. Aber sonst nicht. (Kristin, kosovarische Herkunft)

Kristin fand, dass sich Ausländer nicht nur an die Rechtsordnung anpassen sollten, sondern sich auch in ihrem Sprachgebrauch in der Öffentlichkeit und bei der Knüpfung von Kontakten an der Schweiz orientieren sollten. Sie kritisierte bspw. Eltern, die ihren Kindern nur in der Herkunftsgruppe Freundschaften gewährten.

Auch in Kristins Lebensgestaltung zeigte sich ein Assimilationsbemühen. Sie berichtete, dass sie zu ihrem Herkunftsland kaum noch Bezug hätte und die Sprache

6 Dorina war eine Ausnahme, die allen vier Strategien bzw. keiner ausschliesslich zugeordnet werden konnte. 
verlernt hätte, weil sie in ihrer Familie kaum noch gesprochen würde. Diese Distanz war gewollt, denn sie wollte als Schweizerin anerkannt sein. Auf die Frage, was es ihr bedeute, Schweizerin zu sein, sagte sie:

Nichts, ich gehöre dazu. Ich bin wirklich ein Mitglied von einem Land,
das ich wirklich als meine Heimat empfinde. Weil wenn ich im Kosovo
bin, dann bin ich wie in den Ferien. Mich verbindet nichts damit. Also ich
kann nicht sagen, ich bin Kosovo, ich bin vom Kosovo oder ich bin stolze
Kosovoalbanerin.

Diese Versuche der Grenzüberschreitung und Identifizierung mit Schweizern ging mit einer Abwertung von KosovoalbanerInnen in essenzialistischer Manier einher. Sie argumentierte bspw., dass Frauen im Kosovo im Vergleich zu Männern nichts wert seien oder dass sich Kosovoalbaner in der Schweiz in Schlägereien verwickeln liessen. Kristin meinte sogar, sich für ihre Herkunft zu schämen. Wenn sie neue Leute kennenlerne, so Kristin, nenne sie nur ihren Vornamen, da dieser schweizerisch klänge. Sie vermied auch den Kontakt zu Jugendlichen kosovoalbanischer Herkunft in ihrer Schulklasse und war stolz um jeden Schweizer Kontakt, wie bspw. ihren Freund.

Kristin war überzeugt, dass Ausländer durch Anpassung an die Schweizer Mehrheitsgesellschaft die Grenzlinie überschreiten könnten und als zugehörig betrachtet würden. Sie nahm die Grenzlinie zwar als klar wahr, weshalb das Überschreiten nur durch einen Bruch mit der Herkunftskultur möglich war, weil solche Grenzen nur ein Entweder-Oder zulassen (Alba 2005). Sie empfand die Grenzlinie allerdings als legitim (selbstverschuldet) und durchlässig, wenn man Eigenintiative zeige (Cederberg 2013, 142).

\subsection{Grenzverwischung (boundary blurring) und symbolische Ethnizität}

Teresa, Jasna, Letizia und Nastasia fühlen sich auch als Schweizerinnen, allerdings lehnen sie das Herkunftsland ihrer Eltern nicht so kategorisch ab wie Kristin. Auch wenn sich keine von ihnen als Tschechin, Kroatin oder Ungarin bezeichnen würde, sind sie doch mehr oder weniger stolz, diese «Wurzeln» zu haben und Kontakte mit Verwandten oder Freunden im Heimatland der Eltern zu pflegen. Jasna bspw. war es deshalb wichtig zu präzisieren, dass die Schweiz ihre Heimat ist:

Doch ich bin schon stolz darauf, dass ich von dort komme. Aber ich kann jetzt nicht sagen, dass ich mal nach Kroatien zurückgehen möchte. (...) Ich finde die Schweiz ist meine Heimat. (Jasna, kroatische Herkunft)

Alle vier berichteten, vor allem auf ihre Zweisprachigkeit stolz zu sein (Teresa bezeichnet Tschechisch sogar als ihre Muttersprache), die sie auch an zukünftige Kinder weitergeben möchten. Jasna und Letizia sind zudem der Meinung, dass sie sich aufgrund ihrer typisch kroatischen Mentalität - offen und temperamentvoll - dem Herkunftsland ihrer Eltern verbunden fühlten. Sie wählen klar positiv konnotierte 
Charaktereigenschaften. Teresa erzählte zudem im Interview, dass Leute ihre Herkunft interessant fänden und sich bspw. für den Klang ihrer Sprache interessierten, als Ausländerin habe sie sich daher nie gefühlt:

Das mit dem Ausländer-Sein, ist eigentlich nie so durchgekommen, habe ich das Gefühl, (...) weil es gab eigentlich nie so wirklich eine Situation, wo das ganze irgendwie gespalten wurde. (Teresa, tschechische Herkunft)

Es sind unspektakuläre Momente, in denen ihre Herkunft wichtig wird, weshalb die vier überzeugt waren, noch nie Stigmatisierungen und Diskriminierungen erfahren zu haben. Mit anderen Worten, eine klare Grenzlinie zwischen Ausländern und Schweizern, geschweige damit einhergehenden Ausschluss, hätten sie nie wahrgenommen. Diese Deutung könnte auch ein Versuch sein, sich nicht als Opfer von Ausgrenzung zu verstehen, denn sie berichteten durchaus von Witzen über ihre Herkunft, die sie jedoch als Spass interpretieren und nicht persönlich nähmen.

Ähnlich wie Kristin übertrugen Jasna, Teresa und Letizia ihre eigenen Erfahrungen auch auf andere Einwanderer und fanden es legitim, Integration zu fordern. Ähnlich wie das Luzerner Integrationskonzept betonten sie die Eigenleistung von Einwanderern, so bspw. Jasna: «Ich meine in dem Land, wo ich lebe, soll ich mich auch so benehmen.»

Sie merkten allerdings an, dass Einwanderer «ihre Kultur» nicht vollkommen aufgeben müssten, so argumentierte denn auch Teresa:

[D]iese Integrationsfrage ist immer so aktuell, (...) sie [Ausländer] müssen sich nicht nahtlos einfügen in unsere Gesellschaft, aber eben zum Beispiel die Sprache lernen.

Letizia grenzte sich zudem von MigrantInnen aus dem Balkan ab, die nach ihren Eltern eingewandert seien. Sie würden häufig schlecht Deutsch sprechen, unter sich bleiben und Jüngere eine "Jugosprache» pflegen, mit der sie Aufmerksamkeit erzeugen wollten.

Die vier Frauen vertraten die Ansicht, dass Einwanderer und ihre Nachfahren in der Schweiz dazugehören können, das hänge von ihrer Integrationsbereitschaft ab. Ihre Herkunft müssten sie, im Gegensatz zu Kristin, dennoch nicht verleugnen, die würde als eine Bereicherung angesehen oder toleriert werden. Für sie war die Grenzlinie verschwommen, weshalb sie sich auf beiden Seiten verorteten. Diese symbolische Ethnizität, die sich mit positiven, teils nostalgischen Gefühlen verknüpft, kann sich nur entwickeln, wenn seitens der Mehrheitsgesellschaft nicht mehr mit Stigmatisierungen gerechnet werden muss (Gans 1979; Waters 1990). 


\subsection{Forderung nach gleichberechtigter Anerkennung und Grenzöffnung (boundary shifting)}

Im Gegensatz zu den vorangegangenen Beispielen machten Ardit, Kumrije, Jagos, Ana und Navid auf Stigmatisierungen und Diskriminierungen von Ausländern explizit aufmerksam. Sie nahmen eine Grenzlinie wahr, die sie jedoch anders als Kristin als illegitim empfanden, denn wenn Ausländer sich anpassten (z. B. an Rechtsordnung), so sollten sie auch gleichberechtigt anerkannt werden.

Alle fünf berichteten, in der Schweiz mit Stigmatisierungen konfrontiert zu sein. Ardit erzählte nicht nur von eigenen Mobbingerfahrungen aus seiner Kindheit. Er kritisierte auch die von der Schweizer Volkspartei (SVP) lancierten Abstimmungsinitiativen (z. B. Ausschaffung krimineller Ausländer) und monierte, dass Ausländer teils generalisierend «in einen Topf geworfen» würden. Jagos dagegen berichtete, dass Lehrpersonen aus seiner alten Schule es ihm - einem "Jugo» - nicht gegönnt hätten, eine gute Lehrstelle zu finden. Navid erzählte, vor allem nach dem 11. September mit Vorurteilen konfrontiert worden zu sein, die sich nicht nur gegen sein Herkunftsland («Afghanen seien Terroristen»), sondern auch gegen seine Religion richteten («Islam sei frauenverachtend»).

Von Seiten der Schweiz erwarten alle, dass Ausländer als Ebenbürtige akzeptiert würden; also ihnen die Grenze geöffnet werde. Kumrijes und Navids Einbürgerung war denn auch durch die Vorstellung motiviert, als Eingebürgerte mit mehr Anerkennung rechnen und ggf. einfordern zu können:

Sie [Ältere] müssen das schon akzeptieren, dass ich auch ein Teil der Schweiz bin, dass ich jetzt auch Stimmrecht habe. Und vielleicht würde es ein bisschen was verändern. (Navid, afghanische Herkunft)

Alle empfanden Stigmatisierung und Diskriminierung als ungerecht, weil sie die Schweiz als ihre Heimat ansahen und sich wohlfühlten. Gleichzeitig war ihnen ihre Herkunft viel wichtiger als Teresa, Jasna, Letizia oder Nastasia und anders als Kristin betonten sie, diese nicht verleugnen oder aufgeben zu wollen. Alle berichteten bspw. ihre Muttersprache auch in der Öffentlichkeit zu sprechen. Ihre Forderung nach gleichberechtigter Anerkennung vermischte sich mit der Forderung des Rechtes auf kulturelle Differenz, die essenzialistisch gedeutet wird. Es gebe, so Ardit oder Kumrije, «verschiedene Kulturen» und deshalb wünschten sie sich auch eine/n zukünftige/n PartnerIn, die/der «die ihre» teile. Ana dagegen berichtete, darauf zu bestehen als Portugiesin und nicht als Schweizerin angesehen zu werden.

Allerdings betonten sie, nicht übermässig stolz auf ihre Herkunft zu sein. Ardit und Jagos kritisierten denn auch all jene, die sich nicht integrierten und die Rechtsordnung nicht respektierten (z. B. Raser und Schläger). Sie kannten die stigmatisierenden Fremdzuschreibungen gegenüber ihrer Herkunft und distanzieren sich davon. Kumrije kritisierte die «traditionellen Albaner», die die Freiheiten von 
Frauen nicht akzeptieren würden und identifizierte sich stattdessen mit den in ihren Augen «Modernen», die eine andere Kultur miteinbezogen hätten:

Aber heutzutage sind ja nicht alle so, weil wir hier eben aufgewachsen sind und das ist der Vorteil. Wir haben eine andere Kultur mit einbezogen (...). (Kumrije, kosovarische Herkunft)

Die fünf Jugendlichen forderten ein, dass Einwanderer und ihre Kinder in der Schweiz bedingungslos akzeptiert werden sollten. Für alle fünf war die Grenzlinie zwar wie für Kristin präsent, wurde aber als illegitim empfunden, denn Ausländer sollten gleichberechtigt anerkannt werden und das Recht haben, ihre «Kultur» oder «Religion» auszuleben. Indirekt forderten sie, die Grenze zu öffnen und stützten sich auf das Toleranzpostulat des Luzerner Integrationsleitbildes.

\subsection{Grenzziehung (boundary-making) und Aufwertung der eigenen Herkunft}

Ähnlich wie für Ardit, Navid, Jagos oder Kumrije existierte für Albert, Edi und Admir eine klare Grenze zwischen Ausländern und Schweizern. Den Glauben an eine Öffnung, Verwischung oder Überschreitung teilten die drei jungen Männer mit Vorfahren aus dem Kosovo und Montenegro allerdings nicht, stattdessen identifizierten sie sich mit «ihrer Kultur» und versuchten, darüber eine persönliche Aufwertung zu erzielen.

Alle drei berichteten, dass ihnen «ihre Herkunft» sehr wichtig sei, was vor allem in ihrer Muttersprache zum Ausdruck käme, die sie nicht verlernen wollten und deshalb auch regelmässig in der Familie und mit Freunden sprechen würden. Albert und Edi interessierten sich auch für die Geschichte ihres Landes und hätten mehrheitlich kosovoalbanische Freunde, mit denen man sich aufgrund «der gleichen Kultur» besser verstehen würde.

Auch wenn alle drei in der Schweiz aufgewachsen sind und den Schweizer Pass haben, konnten sie sich nicht vorstellen, sich als Schweizer zu bezeichnen. Die Vorstellung, dass Ausländer allenfalls Papierschweizer werden können, provozierte keine Rebellion, sondern wurde übernommen.

Ich denke, wenn die Eltern aus Montenegro kommen oder aus, ich weiss nicht, es ist halt trotzdem. Zu Hause redet man nicht Deutsch, (...) man wächst halt auch anders auf. Man wird anders von den Eltern erzogen. (Admir, montenegrinische Herkunft)

Albert und Edi bedauerten deshalb im Interview, dass sich einige ihrer Landsleute an die Schweizer Kultur «assimiliert» und ihre Herkunft vernachlässigt oder verloren hätten. Albert ging weiter und fand, dass eine Vernachlässigung der Herkunftskultur mit der Forderung, sich zu integrieren, nicht intendiert sei und zu weit gehe. 
Leute beginnen sich zu assimilieren, was meiner Meinung nach unnötig ist. (...) Du musst deine Herkunft sicher nicht vergessen oder deine Sprache oder was $d u$ bist. (Albert, kosovarische Herkunft)

Im Gegensatz zu den anderen Strategien bezogen sich die drei auch auf ihre Herkunft, um sich auf Kosten der Schweizer Kultur aufzuwerten. Albert und Edi argumentierten im Interview bspw. dafür, dass «die albanische Kultur» solidarischer sei; man zeige mehr Respekt gegenüber Angehörigen bei Todesfällen oder unterstütze Kinder finanziell besser.

Insbesondere Edi und Albert berichteten auch von Stigmatisierungen und Diskriminierungen. Edi erzählte, dass vor allem Frauen negativ reagierten, weil Albaner in ihren Augen Raser oder Schläger seien. Albert dagegen sah sich absolut in einer Opferrolle. Er berichtete nicht nur von eigenen (z. B. Verweigerung der Ausbildungsstelle aufgrund seiner Herkunft), sondern auch von Ereignissen, die ihm zu Ohren gekommen seien (z. B. kosovoalbanische Familie unter Polizeigewalt ungerechtfertigt festgenommen). Seine reaktive Ethnizität (Rumbaut 2008) steht denn auch mit diesen Erfahrungen und Wahrnehmungen im Zusammenhang.

Albert, Edi und Admir forderten genauso wie Ardit usw., dass Einwanderer und ihre Nachfahren ein Recht auf kulturelle Differenz hätten. Aufgrund von Stigmatisierungen und Diskriminierungen war für sie die Grenzlinie genauso präsent. Im Gegensatz teilten sie allerdings nicht den Glauben, dass sich etwas ändern könnte, sodass sie stattdessen die Klarheit der Linie betonten und versuchten, sich über ihre Herkunft aufzuwerten.

\section{Neuenburg: republikanischer Toleranzdiskurs in den Positionierungen der zweiten Generation}

In Neuenburg lassen sich ebenso verschiedene Positionierungen und Strategien im Umgang mit dominanten Grenzlinien ausmachen. Diese unterscheiden sich je nachdem, ob sich die Jugendlichen als "zugehörig» wahrnehmen oder nicht. Erfolgten die Positionierungen in Luzern vorwiegend relational zum dominanten Integrationsparadigma, greifen in Neuenburg beide Gruppen - die "Zugehörigen" resp. «Nicht-Zugehörigen» - auf den republikanischen Toleranzdiskurs gegenüber kultureller Diversität zurück. Innerhalb der zwei Gruppen lassen sich aber verschiedene Strategien identifizieren.

\subsection{Etablierte: Positionierung als «Dazugehörige und SchweizerInnen-Plus» aufgrund verschwommener Grenzen}

Eine erste Gruppe, ItalienerInnen und Portugiesen der zweiten Generation, verstehen sich sowohl als SchweizerInnen als auch als zweite Generation, ähnlich dem zweiten 
Typus in Luzern. Je nach Kontext oder Situation stellen sie die eine oder andere Nationalität in den Vordergrund. Diese Jugendlichen betrachten sich als zugehörig, was ihnen erlaubt, mit ihrer doppelten Zugehörigkeit spielerisch umzugehen.

Etre suisse, ben je vis quand même en Suisse, j'ai le passeport suisse, je suis né en Suisse donc voilà ce que je ressens, quoi. Je suis quand même suisse. (...) [Interviewer: Et tu te sens également italien?] Ouais, dans la vie de tous les jours. J'aime bien quand on me demande de quel pays je viens : "ouais, je suis italien", ça fait toujours plaisir de le dire. (...) Vu que c'est mes racines, voilà, je le ressens toujours. (Luca, italienische Herkunft)

Wir finden hier, was Waters (1990) mit ethnic options bezeichnete, nämlich die Möglichkeit, je nach Kontext zwischen ethnischen resp. nationalen Identitäten zu wählen. Die Herkunft wird in den Praktiken dieser Jugendlichen zu einer Form von symbolischer Ethnizität (Gans 1979) und zu einer nostalgischen Erinnerung. Sie können sich als zugehörig betrachten, weil die Grenzlinie für sie verschwommen ist und sie nicht (mehr) mit Stigmatisierungen rechnen müssen.

So hat Italienisch- und Portugiesisch-Sein in den Augen der Interviewten keine Relevanz für die eigene Partner- oder Freundeswahl, wie etwa Marco (italienische Herkunft) findet: “C'est assez superficiel (...) la nationalité. Je ne trouve ça pas important. » Auch für Luca kommt es allein auf Gefühle für eine Person an. Die Herkunft wird jedoch systematisch im Zusammenhang mit Fussball erwähnt, denn es gilt, für das italienische oder portugiesische Team zu sein. Auch sehen die Jugendlichen kein Problem - im Gegensatz zu den «Nicht-Dazugehörigen» wie unten noch gezeigt wird - in der Schule italienisch oder portugiesisch zu sprechen, wenn sie sich etwa über Dinge austauschen möchten, die nicht alle verstehen sollen (z. B. Luca).

Unter Rückgriff auf ihre symbolische Ethnizität grenzen sich die Jugendlichen zudem klar von den «reinen Schweizern» ab, denn sie würden etwas Zusätzliches, Positives mitbringen. Die italienischen und portugiesischen Jugendlichen (z. B. Clara, Tiago) beschreiben Schweizer als "carré» und verschlossen, Italiener und Portugiesen hingegen als « ouvert». Ähnlich wie Wessendorf (2010) zeigen konnte, profitieren vor allem die ItalienerInnen von der positiven Stereotypisierung einer Italianità, was sie quasi zu SchweizerInnen-Plus macht. Luca sprach im Interview folgendermassen von den Charakteristika von Italienern:

Je pense que quand même, il y a une forme d'élégance, moi je trouve, quand même. (...) Ils sont pas forcément bien habillés en costard, tout ça. (...) Ouais, tout ce qui est accès à la mode, quand même. Il y a des couturiers connus. La mode, c'est quand même une des bases qu'il y à̀ Milan, avec ce qui se passe au niveau mode. (Luca, italienische Herkunft)

Das SchweizerInnen-Plus gilt allerdings für die zweite Generation mit portugiesischen Eltern nur bedingt. Sie können weniger auf solche positiven Stereotypisierungen 
zurückgreifen und erwähnten in den Interviews auch Stigmatisierungen. Tiago klagte sie allerdings nicht (mehr) an:

Avant, ça ménervaient quand ils disaient que les Portugais, ils avaient des poils ou comme ça, mais maintenant je m'en fous. On entend souvent ces blagues. (...) Mais maintenant je trouve ça drôle, et même moi je les dis à mes copains portugais. (Tiago, portugiesische Herkunft)

Auffällig war in den Interviews, dass die Jugendlichen die im öffentlichen Raum dominante Idee einer Toleranz gegenüber «anderen Kulturen» wie auch eine antirassistische Haltung übernehmen.

Moi je pars dans l'idée que toute race est égale (...), je trouve que voilà, tout humain est égal, y a pas besoin d'avoir une fierté particulière. (André, italienische Herkunft)

Allerdings grenzen sich diese Jugendlichen von anderen MigrantInnen v. a. vom Balkan ab. Hierbei stützen sie sich auf ihren Status der "Etablierten», auf ihr Europäisch-Sein, und schaffen eine klare Grenzziehung mittels Kultur, sodass der republikanische Toleranzdiskurs zu einem abrupten Ende kommt:

Mais c'est vrai que c'est quand même des nationalités qui sont assez proches (les Italiens). Donc je pense que ça peut être différent pour des gens qui sont des Balkans, ou encore plus loin. Là, c'est quand même l'Europe. (Clara, italienische Herkunft)

Die Jugendlichen aus Neuenburg mobilisieren dabei ganz ähnliche Elemente wie jene aus Luzern. Die Einwanderer aus dem Kosovo störten im öffentlichen Raum und zettelten bspw. Gruppenschlägereien an.

\section{2 «Nicht-Zugehörige»: Assimilationsstrategien und Aufwertungsstrategien durch republikanischen Toleranzdiskurs um Grenze zu überschreiten oder zu öffnen}

Grundsätzlich andere Positionierungen finden sich unter den Jugendlichen, deren Eltern aus dem Balkan resp. Somalia und Tunesien in die Schweiz einwanderten. Sie äussern eine andere, doppelte Zugehörigkeit, als AusländerIn in der Schweiz und als Bosnier resp. Somalier, ähnlich wie der dritte Typus in Luzern. Sie fühlen sich nicht dazugehörig und sind sich der Grenzziehung gegenüber ihrer Herkunft klar bewusst, so Serif (bosnische Herkunft): "Alors maintenant tu vois le truc c'est qu'en Suisse on est des étrangers, et maintenant en Bosnie on est des étrangers. »

Für Farid ist selbstverständlich, dass er Somalier ist, auch wenn er sich mit der Schweiz identifiziert.

Etre Somalien, ben, c'est mon origine, mon identité, je dirais. Mais comme j'ai quitté la Somalie après trois mois environ, ouais, je suis venu en Suisse, ben, avoir la nationalité suisse c'est aussi, c'est comme une deuxième identité. 
C'est où je vis, ça compte autant pour moi être Suisse qu'être Somalien. (...) Bon, il y a parfois aussi quand on est en Suisse, on se sent plus Somaliens, mais genre, quand on retourne dans notre pays natal on se sent plus Suisses. C'est un peu paradoxal, mais. (Farid, somalische Herkunft)

Alle drei äusserten denn auch, dass sie Erfahrungen mit negativen Stereotypen gemacht hätten, Deuta in der Schule und Ahmed aufgrund seines Namens, der auf ic endet:

Donc quand on cite un nom typiquement comme le mien qui finit en ic, c'est directement une sorte de catégorisation. Donc oui, j'ai déjà ressenti des préjugés un peu. (Ahmed, bosnische Herkunft)

Sie berichten auch von Witzen oder Stigmatisierungen, mit denen sie konfrontiert wurden, insbesondere auch was ihr Muslim-Sein betrifft. Allerdings klagten diese Jugendlichen Witze über ihre Religion oder Herkunft nicht explizit an, sondern machten sich allenfalls darüber lustig. Auf die Frage, ob ihn solche Witze nerven, meinte Farid (somalische Herkunft):

Non, pas du tout. Pas du tout. Ça me fait même rigoler. Ben des blagues, c'est des blagues. (...) Mais ce n'est pas trop de blagues, on délire entre guillemets. Sur les préjugés aussi on fait, par exemple "Les musulmans terroristes, extrémistes". Des choses qu'on sait que ce n'est pas vrai, donc parfois on rigole dessus. Je ne sais pas si c'est bien, mais (...).

Dieser Kategorisierung als AusländerIn begegnen sie mit unterschiedlichen Strategien. Erstens greifen sie den lokal verbreiteten republikanischen Diskurs der kulturellen Bereicherung auf, um sich über ihre Herkunft aufzuwerten. Ahmeds Aussage ist ein besonders prägnantes Beispiel für diese Strategie:

Ben, avant tout c'est un autre pays [Bosnien], un pays que j'aime bien. En tout cas, je n'ai pas honte, je n'ai pas une certaine forme de honte à être, à pouvoir dire que je suis Bosniaque. Pour moi c'est plutôt une fierté, quelque chose qui me démarque des autres. (...) juste on va dire enrichissant au niveau culturel. On a d'autres valeurs que seulement justement des valeurs qu'on nous inculque en Suisse (...) donc cela permet aussi d'avoir d'autres horizons, pas forcément être renfermé qu'avec des amis d'ici. Donc cela permet d'avoir d'autres points de vues, d'autres... vraiment une diversification. (Ahmed, bosnische Herkunft)

Eine Aufwertung ist in diesem spezifischen lokalen Kontext über eine positiv konnotierte (essenzialistisch verstandene) Herkunftskultur möglich. Diese Strategie der Aufwertung durch kulturelle Bereicherung geht zum zweiten mit einer Anpassung an den moralischen Imperativ der Geschlechtergleicheit einher. Die Jugendlichen reagieren auf die gegen sie gerichteten Stereotypisierungen und grenzen sich von 
jenen Vorfahren z. B. aus dem Balkan ab, die sich nicht mit Geschlechtergleichheit identifizieren würden. Serifs Aussage ist in diesem Zusammenhang besonders aufschlussreich:

C'est devenu moderne les Bosniaques, ils sont devenus moderne. Parce qu'à l'époque cétait: la femme elle restait à la maison, elle faisait la cuisine, elle gardait les enfants. Puis c'est l'homme qui amenait le salaire. Bon c'est toujours comme ça, encore maintenant, surtout mes oncles. Mais maintenant on est une nouvelle génération, moi, mes cousins, tout ça, qu'on a plutôt envie que notre femme elle travaille. (...) Maintenant on veut une femme intelligente (...) je sais pas si c'est un peu à cause de la Suisse quion est devenus comme ça. (...) s'est quand même une chiée adaptés à la, à tout quoi. On est devenu modernes. (Serif, bosnische Herkunft)

Eine Aufwertung der «eigenen Kultur» geschieht hier über einen Modernisierungsdiskurs, der sich auf den moralischen Imperativ der Geschlechtergleichheit bezieht und betont, dass sich die Dinge geändert hätten. Sie argumentieren mit Generationenwandel resp. ihrer Anpassung in der Schweiz - zu klar ist diese Grenzlinie, als dass sie sie grundsätzlich in Frage stellen könnten (niemand argumentiert dafür, dass es bspw. im Balkan auch Personen gäbe, die Geschlechtergleichheit leben würden); sie haben weder Ressourcen noch Macht.

Drittens setzen diese Jugendlichen teilweise weitere Vermeidungs- resp. Assimilationsstrategien ein, um die klare Grenzlinie, mit der sie konfrontiert sind, zu überschreiten und sich auf der anderen, der "guten", Seite zu verorten. Sie vermeiden es, bspw. in der Öffentlichkeit in ihrer Herkunftssprache zu sprechen oder ihre Religion zu thematisieren - ganz im Gegensatz zum ersten Typus. Clément findet es wichtig, in Anwesenheit anderer nicht arabisch zu sprechen:

C'est bien avec des amis, quand on est tous ensemble, là on parle (...). Mais par exemple, sil y a des gens, par exemple si vous êtes avec moi et qu'il y a un pote arabe ici, un autre là, qu'on est trois de la même langue, moi je parlerai français, comme ça vous pouvez comprendre ce qu'on dit. Comme ça la personne elle se fait pas des idées dans la tête, parce quion rigole ou comme ça, de se dire "Ouais, ils se moquent de moi». Ça, j’ai toujours respecté, les gens autour de moi qui parlent français, et si j’ai des amis arabes, je parle français (Clément, tunesisch-französische Herkunft)

Ähnliches berichtet Farid, wenn er vermeidet, somalisch zu sprechen: «A l'école, ici j'en connais [des autres Somaliens], mais je parle le français. "

Schliesslich finden sich unter diesen «Nicht-Zugehörigen» zusätzlich Grenzöffnungsstrategien. Die fünf schreiben sich mit Rückgriff auf ihre Herkunft in den republikanischen Toleranzdiskurs gegenüber kultureller Diversität ein und betonen 
in den Interviews immer wieder, wie tolerant sie ihrerseits seien, eine Eigenschaft die für ihre Herkunftsgruppe typisch sei:

[Etre bosniaque] c'est quelque chose de fort. On a des liens qui sont hyper-fort. On accepte tout le monde (...). Moi je suis ami avec tout le monde hein. Je refuse personne, je suis pas raciste. (Séfid, bosnische Herkunft)

All diese Strategien sind Versuche, die klare Grenzziehung zwischen ihnen als den "Ausländern» und den «anderen» aufzuweichen bzw. zu überschreiten. Sie wissen, dass sie nicht zu den Etablierten gehören, versuchen aber, durch Bestätigung der dominanten Diskurse - Toleranzdiskurs und Geschlechtergleichberechtigung - die Grenzlinie zugunsten ihrer Herkunftsgruppe zu verschieben und sich auf der "guten» Seite zu positionieren.

\section{Diskussion}

Unsere Analyse knüpft an zentrale Erkenntnisse der Integrationsforschung an. Wir gingen der Frage nach, wie sich die zweite Generation gegenüber ethno-nationalen Grenzlinien und Fremdzuschreibungen positioniert, und zeigten, dass auch in der Schweiz multiple Inkorporationswege, aus unserer Perspektive formuliert multiple Vorstellungen über Zugehörigkeit und Fremdheit, existieren. Der empirische Vergleich der zweiten Generation aus Luzern und Neuenburg machte deutlich, dass diese im Kontext "kultureller Repertoires», die wir anhand der lokalen Integrationspolitik und -diskurse analysierten, verstanden werden müssen. «Kulturelle Repertoires» definieren Zugehörigkeiten quasi vor und kanalisieren Strategien, wie mit Grenzziehungen umgegangen wird.

Insbesondere Alba (2005) hat auf die Institutionalisierung von Grenzlinien in gesellschaftlichen Schlüsselbereichen (z. B. Staatsbürgerschaftskonzept, Einwanderungs- und Integrationspolitik) hingewiesen. Sie bestimmen, welche Zugehörigkeiten Einwanderern und ihren Nachfahren zugestanden werden und wie sie sie im Lebensalltag verhandeln können (Neckel und Sutterlüty 2008, 17). Sind Grenzlinien stark institutionalisiert (bright boundaries), d. h., historisch, politisch, rechtlich, sozialstrukturell oder kulturell in einen Kontext eingeschrieben, so wissen Einwanderer, auf welcher Seite der Linie sie sich befinden (Alba 2005, 41). In dem Fall werden Stigmatisierungen eher mit Identifikation mit der bessergestellten Gruppe oder Aufwertung der eigenen Gruppe begegnet. Sind Grenzlinien kaum institutionalisiert (blurred boundaries), so ist die Zugehörigkeit zu einer der beiden Seiten unklar und Grenzverwischungen und -verschiebungen leichter vorstellbar und durchsetzbar. Diese kontextspezifischen Überlegungen bestätigen sich in unserem empirischen Vergleich beider Kantone, allerdings müssen sie ausdifferenziert werden. Zum einen sind die gewählten Positionierungen vielfältiger, wie von Alba 
(2005) konzeptualisiert, und zum anderen zeigt sich in den Positionierungen auch das regionale kulturelle Repertoire, auf das die Jugendlichen in ihren Grenzziehungsstrategien zurückgreifen.

In Luzern und Neuenburg gibt es Nachfahren von Einwanderern, die sich als «zugehörig» fühlen. Sie haben die Möglichkeit multiple, ethnische oder nationale Zugehörigkeit situativ zu wählen, denn die Grenzziehung gegenüber ihrer Herkunft ist verwischt. Ethnizität hat einen symbolischen Charakter. Sie sind stolz auf ihre Herkunft, die sie durchweg positiv - im Vergleich zum «Schweizerisch-Sein» - aufladen. Auffällig ist, dass diese Jugendlichen in Neuenburg einen starken Toleranzdiskurs gegenüber anderen «Kulturen» und eine anti-rassistische Haltung vertreten - Elemente, die in Luzern nicht auftauchten. Diese «Dazugehörigen» grenzen sich in beiden Kontexten von anderen MigrantInnen, vor allem vom Balkan, ab. Während in Luzern die Abgrenzung durch Bezug auf die Integrationsforderung und die nötige Eigeninitiative erfolgt, so wird die Grenzlinie in Neuenburg über «kulturell anders sein» gerechtfertigt. Kein Zufall ist, dass diese Jugendlichen europäische Vorfahren haben. Hier zeichnet sich eine institutionalisierte Grenzlinie zwischen europäischen und nicht-europäischen Einwanderern ab. Aufgrund des Freizügigkeitsabkommens bspw. sind nur Letztere Integrationsforderungen unterworfen.

Bei der zweiten Generation, die sich gegenüber bright boundaries positioniert, v.a. solche mit Eltern aus dem Balkan, aber auch aussereuropäischen Ländern, finden sich sowohl Assimilationsstrategien (boundary crossing), d. h. Versuche, sich mit der bessergestellten Gruppe zu identifizieren, wie auch Aufwertungsstrategien bezüglich der eigenen Gruppe - wie von Alba beschrieben. Allerdings sind die Übergänge fliessend, können kombiniert werden und erneut: Sie sind eingebettet in lokale Diskurse. Assimilationsstrategien erfolgen in Luzern gekoppelt an eine Distanzierung von und Abwertung der «eigenen Gruppe», eine quasi «entwederoder» Strategie, wobei die dominante Integrationsforderung sehr präsent ist. In Neuenburg finden wir in den Assimilationsstrategien weder Integrationsforderungen, noch eine Abwertung der «eigenen Gruppe» - vermutlich erneut aufgrund des vorherrschenden Toleranzdiskurses. Zudem lassen sich in Luzern im Kontext dieser «Entweder SchweizerIn oder AusländerIn»-Konfiguration sowohl Forderungen nach gleichberechtigter Anerkennung (auch kultureller Spezifitäten) ausmachen - im Sinne einer Grenzöffnung - als auch Strategien, die die eigene Herkunft reaktiv und in Abgrenzung aufwerten - d. h. reaktive Ethnisierungen. In Neuenburg fehlen diese Strategien, die «Nicht-Dazugehörigen» versuchen stattdessen, sich auf der «guten Seite» der Grenzlinie zu platzieren, indem sie auf die Idee der kulturellen Bereicherung durch ihre Herkunft zurückgreifen oder mit einem Modernisierungsdiskurs argumentieren. Zudem findet sich hier erneut eine auf Toleranz ausgerichtete, antirassistische Haltung.

Mit anderen Worten: Die zweite Generation ist in beiden Kantonen mit einer Grenzlinie zwischen Schweizern und Einwanderern konfrontiert. Essenzialistisch 
definierte Differenz wird allerdings in Neuenburg grösstenteils als positive Bereicherung angesehen, während in Luzern eine negative Aufladung stärker präsent ist. Hier spiegeln sich unterschiedlich institutionalisierte Vorstellungen über kulturelle Diversität und Integration wider, mit der Folge, dass die zweite Generation in Luzern mit der Grenzlinie stärker konfrontiert ist als in Neuenburg und ihre Positionierungen gegenüber Fremdzuschreibungen unterschiedlich kanalisiert werden. Vor diesem Hintergrund fordern einige Luzerner Jugendliche gezielt eine gleichberechtigte Anerkennung oder das Recht auf kulturelle Differenz, um ihre Zugehörigkeit zur Schweiz einzuklagen; Diskurse also, auf die Jugendliche im Kontext Neuenburgs bereits bauen können. Die Relevanz des Integrationsthemas bzw. die Idee von Toleranz und kultureller Bereicherung in den Positionierungen der zweiten Generation sagt letztlich etwas über die Art der Grenzlinie aus und macht deutlich, welche Formen von Zugehörigkeit der zweiten Generation zugestanden wird bzw. eingefordert werden müssen.

\section{Literaturverzeichnis}

Alba, Richard. 2005. Bright vs. blurred boundaries: Second-generation assimilation and exclusion in France, Germany, and the United States. Ethnic and Racial Studies 28(1): 20-49.

Alba, Richard D. und Nee Viktor. 1997. Rethinking assimiliation theory for a new era of immigration. International Migration Review 31(4): 826-874.

Amelina, Anna und Thomas Faist. 2012. De-naturalizing the national in research methodologies: Key concepts of transnational studies in migration. Ethnic and Racial Studies 35(10): 1707-1724.

Aumüller, Jutta. 2009. Assimilation. Kontroversen um ein migrationspolitisches Konzept. Wetzlar: transcript.

Barth, Frederik. 1969. Ethnic Groups and Boundaries: The Social Organization of Culture Difference. London: Allen \& Unwin.

Bolzman, Claudio, Rosita Fibbi und Marie Vial. 2003. Secondas - Secondos. Le processus d'intégration des jeunes adultes issus de la migration espagnole et italienne en Suisse. Zürich: Seismo Verlag.

Bommes, Michael und Dietrich Thränhardt. 2010. National Paradigms of Migration Research. Osnabrück: Institut für Migrationsforschung und Interkulturelle Studien.

Brubaker, Rogers. 1992. Citizenship and Nationhood in France and Germany. Cambridge, MA: Harvard University Press.

Brubaker, Rogers, Mara Loveman und Peter Stamatov. 2004. Ethnicity as cognition. Theory and Society 33: 31-64.

Cederberg, Maja. 2013. Public discourses and migrant stories of integration and inequality: Language and power in biographical narratives. Sociology online first, 6. Juni 2013.

Charmaz, Kathy. 2001. Qualitative interviewing and grounded theory analysis. S. 675-694 in Handbook of Interview Research. Context and Methods, hrsg. von Jaber F. Gubrium und James A. Holstein. Thousand Oaks: Sage Publications.

Crul, Maurice und Jens Schneider. 2013. Second-generation migrants: Europe and the United States. S. 1-5 in The Encyclopedia of Global Human Migration, hrsg. von Immanuel Ness und Peter Bellwood. UK: Blackwell Publishing Ltd. 
Dahinden, Janine. 2008. Deconstructing mythological foundations of ethnic identities and ethnic group formation: Albanian-speaking and new Armenian immigrants in Switzerland. Journal of Ethnic and Migration Studies 34(1): 55-76.

Dahinden, Janine. 2011. «Kulturelle Vielfalt»? Grenzziehungen mittels «Kultur» im Kontext von Migration und Integration. S. 33-46 in Von der Deklaration zur Umsetzung - Schutz und Förderung der kulturellen Vielfalt in der Schweiz. Akten der Tagung vom 25. Januar 2011, Zürich, hrsg. von SAGW. Bern: Schweizerische Akademie der Geistes- und Sozialwissenschaften.

Dahinden, Janine. 2012. Transnational belonging, non-ethnic forms of identification and diverse mobilities: Rethinking migrant integration? S. 117-128 in Migration: Interdisciplinary Perspectives, hrsg. von Michi Messer, Renée Schroeder und Ruth Wodak. Wien: Springer.

Duemmler, Kerstin. 2015. Symbolische Grenzen - Zur Reproduktion sozialer Ungleichheit durch ethnische und religiöse Zuschreibungen. Bielefeld: Transcript.

Elias, Norbert und John L. Scotson. 1994 [1965]. The Established and the Outsiders. A Sociological Enquiry. London: Sage.

Fibbi, Rosita, Mathias Lerch und Philippe Wanner. 2006. Unemployment and discrimination against youth of immigrant origin in Switzerland: When the name makes the difference. Journal of International Migration and Integration 7(3): 351-366.

Gans, Herbert J. 1979. Symbolic ethnicity: The future of ethnic groups and cultures in America. Ethnic and Racial Studies 2(1): 1-19.

Gesundheits- und Sozialdepartement. 2013. Kantonales Integrationsprogramm 2014-2017. Spezifische Integrationsförderung als Verbundaufgabe Bund - Kantone. Luzern: Kanton Luzern.

Glaser, Barney und Anselm L. Strauss. 1967. The Discovery of Grounded Theory. Chicago: Aldine.

Jenkins, Richard. 2008. Rethinking Ethnicity: Arguments and Explorations. London: Sage.

Kelle, Udo und Susann Kluge. 2010. Vom Einzelfall zum Typus. Wiesbaden: VS Verlag.

Lamont, Michèle und Nissim Mizrachi. 2012. Ordinary people doing extraordinary things: Responses to stigmatization in comparative perspective. Ethnic and Racial Studies 35(3): 365-381.

Lamont, Michèle und Virag Molnar. 2002. The study of boundaries in the social sciences. Annual Review of Sociology 28: 167-195.

Menet, Joanna und Nicole Wichmann. 2012. Die schweizerische Integrationspolitik zwischen Fördern und Fordern. In: Integration per Unterschrift? Zweckmässigkeit von Integrationsvereinbarungen im Migrationsrecht. Tagungsdokumentation. Konstanzer Wissenschaftsforum: 13-19.

Neckel, Sighard und Ferdinand Sutterlüty. 2008. Negative Klassifikationen und die symbolische Ordnung sozialer Ungleichheit. S. 15-25 in Mittendrin im Abseits. Ethnische Gruppenbeziehungen im lokalen Kontext, hrsg. von Sighard Neckel und Hans-Georg Soeffner. Wiesbaden: VS Verlag.

Phillips, Anne. 2010. What's wrong with Essentialism? Scandinavian Journal of Social Theory 11(1): 47-60.

Portes, Alejandro, Maria Patricia Fernandez-Kelly und William Haller. 2009. The adaptation of the immigrant second generation in America: A theoretical overview and recent evidence. Journal of Ethnic and Migration Studies 35(7): 1077-1104.

Portes, Alejandro und Rubén G. Rumbaut. 2005. Introduction: The second generation and the children of immigrant's longitudinal study. Ethnic and Racial Studies 28: 983-999.

Regierungsrat. 2001. Über die Ausländer- und Integrationspolitik des Kantons Luzern. Luzern: Regierungsrat.

Rumbaut, Rubén G. 2008. Reaping what you sow: Immigration, youth, and reactive ethnicity. Applied Developmental Science 12(2): 108-111.

Schiffauer, Werner, Gerd Baumann, Riva Kastoryano und Steven Vertovec 2002. Staat-Schule-Ethnizität. Münster: Waxmann. 
Schneider, Jens und Maurice Crul. 2010. New insights into assimilation and integration theory: Introduction to the special issue. Ethnic and Racial Studies 33(7): 1143-1148.

Service de la cohésion multiculturelle. 2009. Fondements et principes de la République et Canton de Neuchâtel. Neuchâtel: Canton de Neuchâtel, http://www.ne.ch/autorites/DEAS/COSM/chartecitoyennete/Documents/Charte/mementoA4FRA.pdf, (24.08.2015).

Service de la cohésion multiculturelle. 2013. Programme cantonal d'intégration (PIC). Neuchâtel: Canton de Neuchâtel, http://www.ne.ch/autorites/DEAS/COSM/Documents/PIC.pdf (24.08.2015).

Simon, Patrick. 2003. France and the unkown second generation: Preliminary results on social mobility. International Migration Review 37: 1091-1119.

Swidler, Ann. 1986. Culture in action: Symbols and strategies. American Sociological Review 51(2): 273-286.

Vasta, Ellie. 2007. From ethnic minorities to ethnic majority policy: Multiculturalism and the shift to assimilation in the Netherlands. Ethnic and Racial Studies 30(5): 713-740.

Waters, Mary C. 1990. Ethnic Options. Choosing Identitites in America. Berkeley, Los Angeles, London: University of California Press.

Weber, Max. 1922 [1980]. Wirtschaft und Gesellschaft. Tübingen: Mohr.

Wessendorf, Susanne. 2010. Local attachments and transnational everyday lives: Second-generation Italians in Switzerland. Global Networks 10(3): 365-382.

Wimmer, Andreas. 2008. Elementary strategies of ethnic boundary making. Ethnic and Racial Studies 31(6): 1025-1055.

Wimmer, Andreas. 2013. Ethnic Boundary Making. Institutions, Power and Networks. Oxford: Oxford University Press.

Wimmer, Andreas und Nina Glick Schiller. 2002. Methodological nationalism and beyond: Nation-State building, migration and the social sciences. Global Networks 2(4): 301-334.

Zhou, Min. 1997. Segmented assimilation: Issues, controversies, and recent research on the new second generation. International Migration Review 31(4): 975-1008.

Zolberg, Aristide und Long Lit Woon. 1999. Why Islam is like Spanish: Cultural incorporation in Europe and the United States. Politics \& Society 27(1): 5-38. 


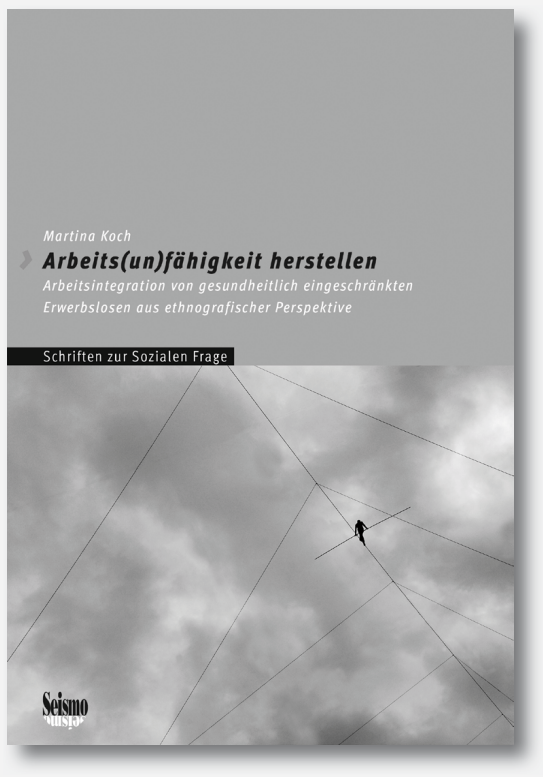

Wie in den meisten entwickelten Ländern ist auch in der Schweiz zu beobachten, dass zunehmend Menschen mit gesundheitlichen Einschränkungen und «Behinderungen» aktiviert und beruflich integriert werden sollen. Die immer stärker vorgetragene politische Forderung, Menschen mit gesundheitlichen Einschränkungen beruflich einzugliedern, stellt die einschlägigen Institutionen und Fachkräfte vor grosse Herausforderungen. In der Folge vervielfältigen sich entsprechende Praktiken, während die Instrumente und Methoden zur Feststellung von Arbeits(un)fähigkeit verfeinert werden. Anhand einer Ethnografie in zwei kantonalen Arbeitsintegrationsagenturen geht diese Studie der Frage nach, wie die un-

\section{Martina Koch}

Arbeits(un)fähigkeit herstellen

Arbeitsintegration von

\section{gesundheitlich einge-}

schränkten Erwerbslosen aus ethnografischer Perspektive

Reihe

«Schriften zur Sozialen Frage»

268 Seiten, ISBN 978-3-03777-155-6, SFr. 38.-

tersuchten Organisationen die Arbeits(un)fähigkeit ihrer KlientInnen konstruieren und bearbeiten. Im Fokus stehen die organisationalen Problematisierungs- und Bearbeitungsstrategien und dahinterstehende Logiken.

Martina Koch, Dr. rer. soc., promovierte an der Universität St. Gallen. Sie ist Soziologin und arbeitet als wissenschaftliche Mitarbeiterin an der Hochschule für Soziale Arbeit der Fachhochschule Nordwestschweiz (FHNW), wo sie in den Bereichen Sozialstaat und Sozialpolitik, Erwerbslosigkeit und Arbeitsintegration sowie Diversität in der öffentlichen Verwaltung forscht. 